\title{
Simultaneous classification and location of volcanic deformation in SAR interferograms using deep learning and the VolcNet database
}

\author{
M. Gaddes ${ }^{\mathrm{a}}$, A. Hooper ${ }^{\mathrm{a}}$, F. Albino ${ }^{\mathrm{b}}$ \\ ${ }^{a}$ COMET, University of Leeds, UK. \\ ${ }^{b}$ School of Earth Sciences, University of Bristol.
}

\begin{abstract}
This manuscript is an EarthArXiv preprint that has been through one round of peer review after being submitted for publication in Remote Sensing of Environment.
\end{abstract}

With the evolution of InSAR into a tool for active hazard monitoring, through its ability to detect ground deformation with low latency, new methods are sought to quickly and automatically interpret the large number of interferograms that are created. In this work, we present a convolutional neural network (CNN) that is able to both classify the type of deformation, and to locate the deformation within an interferogram in a single step. We achieve this through building a "two headed model", which is able to return both outputs after one forward pass of an interferogram though the network, and so does not require the use of a sliding window approach for localisation. We train our model by first creating a large dataset of synthetic interferograms 
which feature labels of both the type and location of any deformation, and we release the Python3 code for this as a package named SyInterferoPy. We find that our model's performance is improved through the inclusion of just a small amount of augmented real Sentinel-1 data, and retrain our model accordingly. We also release this set of labelled training data as a database named VolcNet. When building models of this type, it is common for some of the weights within the model to be transferred from other models designed for different problems. Consequently, we also investigate how to best organise interferograms such that the filters learned in models such as VGG16 are sensitive to the signals of interest in interferograms, but find that using different data in each of the three input channels significantly degrades performance when compared to the simple case of repeating wrapped and unwrapped phase across each channel. This implies that the inclusion of supplementary data, which we expect should improve the ability to distinguish deformation from noise, requires training of a network from scratch.

Keywords: volcano monitoring, CNN, InSAR, VGG16, VolcNet, SyInterferoPy

\section{Introduction}

2 In recent years, work to extend volcano monitoring to all of the world's $3 \sim 1400$ subaerial volcanoes has resulted in the application of several machine 4 learning methods to ground deformation maps produced by interferometric 5 synthetic aperture radar (InSAR). Work presented in Anantrasirichai et al. 6 (2018, 2019a b) and Valade et al. (2019) has used convolutional neural net7 works (CNNs) to determine if individual interferograms contain deformation, 
whilst time series have been used by Gaddes et al. (2018) to detect signs of unrest and by Sun et al. (2020) to detect subtle deformation signals. However, in both of the examples detailed above, each algorithm demonstrates very limited knowledge of the diverse types of deformation that may be measured at volcanoes. The algorithm presented in Anantrasirichai et al. (2019a) assigns all data containing deformation to one label, whilst the algorithm presented in Gaddes et al. (2018) alerts users to changes in the signals present, but does not identify the type of deformation present. Consequently, we seek to improve upon these approaches by developing a CNN that is able to differentiate between different types of deformation, and to detect the spatial extent of it.

Figure 1A shows the hierarchy of computer vision object/signal identification methods. The algorithm presented in Anantrasirichai et al. (2018) contains a model that performs classification and, by breaking larger images into smaller tiles that are each classified, the algorithm as a whole is able to perform localisation. This approach has the limitation that the deep learning model used in this algorithm does not need to learn how to determine the location or size of the object (or signal) of interest, and at a more fundamental level, remains a classification and not localisation model. However, in the field of computer vision, CNNs have been developed that are able to perform both classification and localisation on images that contain either single or multiple objects. The location of an object is either indicated through encompassing it in a rectangle (e.g. localisation or object detection, Simonyan and Zisserman (2014); Redmon et al. (2016) ) or, in more complex algorithms, indicating the exact outline of an object by identifying which pixels comprise it (e.g. 
instance segmentation, He et al. (2017)). These approaches should provide more detailed information on the spatial extent of a signal of interest than a classification model that is repeatedly used on different areas of the representation. Consequently, we endeavour to advance the state of the art through developing a CNN that is able to both localise deformation within an interferogram, and to classify different types of deformation (the hierarchy of which we show in Figure 1B).

When constructing a CNN to perform both classification and localisation with data derived from SAR satellites, a new CNN could be designed before all the parameters within it are trained. However, this approach has the risk of failing to utilise the successful applications of CNNs to other computer vision problems. When reviewing methods aimed at incorporating previous successful models, the two disparate parts of a CNN must be considered. An example CNN is shown in Figure $1 \mathrm{C}$, in which the convolutional part comprises of filters that are convolved across an image to extract deep representations, whilst downsampling is performed simultaneously to reduce the spatial extent of an image. In the case of the example network show in in Figure 1C, a three channel (colour) image of size $(224 \times 224 \times 3)$ pixels is transformed into a spatially smaller but deep $(7 \times 7 \times 512)$ representation by this process. In the second part, this $3 \mathrm{D}$ representation is flattened into a vector (which in this example would be of size $(7 \times 7 \times 512=25088)$, before a traditional neural network comprising of interconnected neurons is used to create the desired model outputs. The size of the last layer of this second part is dependent on features such as the number of different classes present in the data and, in this example case with two neurons in the last 
layer, would be used in a case in which there were only two different classes. Consequently, when using an existing model on a new problem, any change in the number or type of output classes will require changing the fully connected part of the network. Therefore, it is common to retain the convolutional layers (i.e. part one of the model) and design a new fully connected network (i.e. part two of the model) that outputs the classes required by the new problem. However, this approach still requires the training of a CNN that is likely to contain tens of millions of parameters, which will be both computationally expensive, and require a large volume of training data. AlexNet, a previously state-of-the-art image classification CNN (named after one of the designers, Alex Krizhevsky), has 60 million parameters, was trained on 1.2 million images, and even when implemented on GPUs took around one week to train (Krizhevsky et al. 2012). Therefore, a common approach termed transfer learning is to retain both the structure and weights of the initial convolutional layers, and to train only the fully connected part of the network. This approach was successfully used by Anantrasirichai et al. (2018), who used the structure and weights of AlexNet, but created their own fully connected classifier to output whether an interferogram contained deformation or not.

The weights learned in the convolutional filters of a CNN are of great importance to a network's ability to detect features, as the filters must be sensitive to the patterns that these features present in an image. As networks such as AlexNet (Krizhevsky et al., 2012) and VGG16 (Simonyan and Zisserman (2014), named after the University of Oxford Visual Geometry Group) were originally developed to compete in the ImageNet competitions (Deng et al. 2009), the filters have been trained to detect the type of features present in 
natural images (e.g. photographs of a person, or car). When performing transfer learning, it is these filters that must be sensitive to the patterns presented in a deformation signal if the network is to correctly classify and locate it. However, as interferograms can be expressed in differing formats we also seek to explore which of these formats allows for the filters in models trained on natural images to excel.

\section{Classification with different data formats}

As the most common CNNs for computer vision are trained on images comprising of a channel for each of the red, green, and blue values for each pixel, other data that are to be used with the network must also be three channel. However, when considering an image of interferometric phase, these images contain only a single value for each pixel, and so consist of only one channel, and are analogous to a greyscale image. This difference in the number of channels can be circumvented through duplicating the one channel interferogram in each of the three input channels of a CNN, but in this section of our study we wish to determine if this approach can be improved upon.

When two SAR images are combined to form a single interferogram, the resulting image is a $2 D$ array of complex numbers (Hanssen, 2001). Whilst the magnitude of each of these complex numbers relates to the underlying brightness and coherence of a given pixel, it is common for only the argument to be displayed, as these phase values can be used to infer ground movement. However, the phase values of an interferogram are wrapped in the range $[-\pi, \pi]$ as only the fractional part of the phase value can be measured, but 
A)

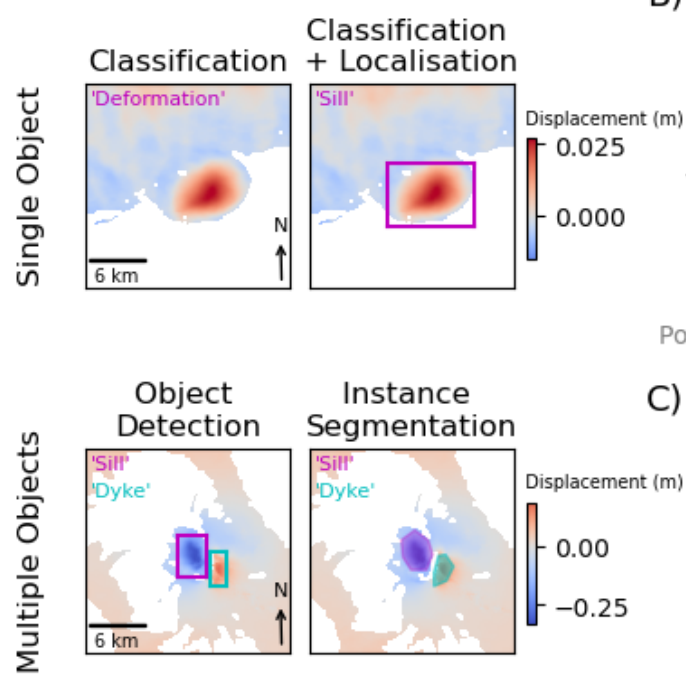

B)

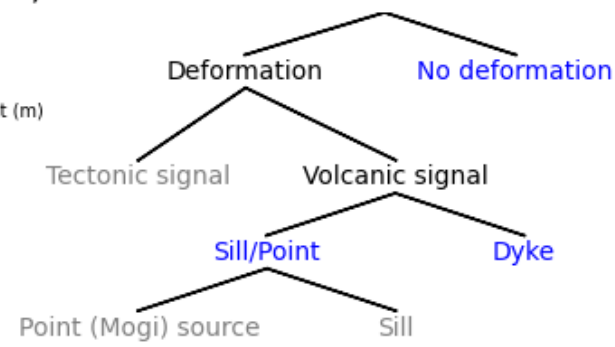

C)

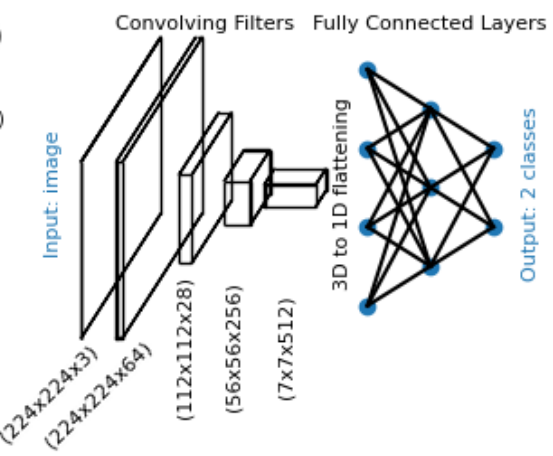

Figure 1: A) Introduction to the hierarchy of computer vision object/signal identification methods. The upper and lower rows show 12 day descending Sentinel-1 interferograms of Sierra Negra and Wolf volcano (Galapagos Archipelago, Ecuador), respectively. The Sierra Negra interferogram contains only one signal (an inflating sill), whilst the Wolf interferogram contains two signals (a deflating sill and an opening dyke). B) Proposed hierarchy for signals of interest in interferograms at volcanic centres. We propose a model that is able to classify interferograms into one of the three classes shown in blue: "no deformation", "Dyke", and ""Sill/Point". We envisage that future studies may add further classes which we mark in grey, such as those that differentiate between sills and point sources. C) Overview of a traditional convolutional neural network (CNN), showing how convolving filters and downsampling create a small but deep representation of an image $((224 \times 224 \times 3)$ to $(7 \times 7 \times 512))$, which is then flattened and passed through a traditional neural network. 
this ambiguity can be estimated to produce an unwrapped interferogram (Chen and Zebker, 2001). We postulate that in addition to the use of either wrapped or unwrapped data duplicated to fill three channels, the original complex numbers of an interferogram could be used in two channels, and so allow the network to use interferometric amplitude as an indicator of the reliability of the phase.

However, we can also consider external data to feed into the CNN. When a human observer interprets an interferogram, they are likely to use data such as a digital elevation model (DEM) as this can be used to help determine if a signal is due to deformation, or due to a topographically-correlated atmospheric phase screen. This problem is of particular importance at stratovolcanoes, as the cones typical of these volcanoes can be several kilometres high, and therefore be capable of creating large and spatially stationary signals in interferograms. The body of literature that covers the application of InSAR to volcanic deformation is replete with studies that consider which of the two mechanisms are responsible for the observed signals, and examples include Beauducel et al. (2000); Rémy et al. (2015); Yip et al. (2019). When considering previous attempts at the automatic detection of deformation signals in Sentinel-1 interferograms, Anantrasirichai et al. (2019a) also reported that many of the false positives recovered by their algorithm were caused by signals correlated with topography. Consequently, we postulate that the inclusion of a DEM in the inputs to our CNN will improve its ability to differentiate between deformation signals and atmospheric signals that are correlated with topography, and therefore seek to investigate its use as an input into a multichannel model. 
To perform this analysis, we first synthesise a dataset of labelled interferograms. To achieve this, we have created an open source Python3 package named SyInterferoPy, which we make freely available to the community via GitHub: https://github.com/matthew-gaddes/SyInterferoPy. The collection of enough labelled data to train a CNN is commonly time consuming or expensive, and we find that the addition of localisation labels to our data makes it more time consuming than in previous studies. Additionally, due to the large number of data that are required to train CNNs and our expansion to classification of different types of deformation, procuring enough real data to do this may be not possible. Consequently, we perform this analysis using only synthetic data. Following the hierarchy proposed in Figure 1B, we create interferograms that contain either no deformation, deformation due to an opening dyke, or deformation due to a sill or point source. These sources were chosen after reviewing the database of volcanic deformation events measured using InSAR in Biggs et al. (2014) as we believe they cover the majority of the observed signals that are of importance for volcano monitoring (i.e. we disregard signals due to processes such as the cooling of lava flows). We model the dykes as vertical dislocations with uniform opening in an elastic half space (Okada, 1985) with strikes in the range $\left[0,359^{\circ}\right]$, dips in the range $\left[75,90^{\circ}\right]$, openings in the range $[0.1,0.7] \mathrm{m}$, top depths in the range $[0,2] \mathrm{km}$, bottom depths in the range $[0,8] \mathrm{km}$, and lengths in the range $[0,10] \mathrm{km}$. We model the sill/point sources as horizontal dislocations with uniform opening in an elastic half space (Okada, 1985) with strikes in the range $\left[0,359^{\circ}\right]$, dips in the range $\left[0,5^{\circ}\right]$, openings in the range $[0.2,1] \mathrm{m}$, depths in the range $[1.5,3.5]$ $\mathrm{km}$, and widths and lengths in the range $[2,6] \mathrm{km}$. It should be noted that 
our proposed hierarchy of volcanic deformation signals also includes processes that could be modelled as a point pressure source (commonly referred to as a "Mogi" source (Mogi, 1958)) within the sill/point category, but given that we do not envisage that a deep learning model using satellite data from only one look angle (i.e. ascending or descending) would be able to differentiate between these two models, we generate our synthetic data using only one of them for simplicity.

These deformation patterns are then combined with a topographically correlated atmospheric phase screen (APS), and a turbulent APS, which we discuss generating in more detail in Gaddes et al. (2018). We calculate the topographically correlated APS using the Shuttle Radar Topography Mission (SRTM) 90m DEM (Farr et al., 2007), and use the coastline information contained within the product to mask areas of water. We also synthesise areas of incoherence within our interferograms, which we mask in order for our synthetic interferograms to be as similar as possible to the Sentinel-1 interferograms automatically created by the LiCSAR processor (Lazeckỳ et al. 2020). Figure 2 shows the results of mixing these different elements to create our synthetic interferograms, and the range of sizes of deforming regions that the different deformation model parameters produce (e.g. Interferogram 2 versus Interferogram 3).

This process creates unwrapped data, which can be converted to wrapped data through finding modulo $2 \pi$ of the unwrapped phase. However, to synthesise both the real and imaginary part of a complex interferogram requires knowledge of both the brightness of a pixel and its phase. To achieve this, we again use the SRTM DEM, and calculate the intensity of reflected electro- 
magnetic radiation at the angles of incidence used by the Sentinel-1 satellites $\left(29.1-46.0^{\circ}\right)$, before adding speckle noise, and calculating the interferometric amplitude between two images (i.e. the product of the two amplitudes). As inputs to CNNs that are to be trained using transfer learning must be rescaled to the inputs used in the original training data, we use only relative values in the range $[(-1),-1]$ for the synthetic intensities. With knowledge of the modulus (relative intensity) and argument (wrapped phase) of each pixel of our synthetic interferogram, the real/imaginary components are simply the products of the modulus and cosine/sine of the argument, respectively. Figure 3 shows five different ways we can represent an interferogram using the three channels available.

The CNN we build to classify the synthetic interferograms uses the five convolutional blocks of VGG16 (Simonyan and Zisserman, 2014), with our own fully connected network after this. This network was chosen as, when used in the field of computer vision for classifying natural images, it outperformed older models such as AlexNet (Simonyan and Zisserman, 2014), which is used in the algorithm presented in Anantrasirichai et al. (2018). Figure 4B shows an overview of the model, in which interferograms of shape $(224 \times 224 \times 3)$ are passed through the five convolutional blocks of VGG16 to create a tensor of shape $(7 \times 7 \times 512)$. This is flattened to make a vector of size 25,088 , before being passed through fully connected layers of size 256, 128, and an output layer of size three (i.e., dyke, sill/point, or no deformation). The localisation output shown in the figure is not used in our preliminary exploration of which channel format to use (Section 2), but is used in Section 3. To produce a set of outputs that can be used as probabilities, we use a softmax 
activation for the last layer (Bridle, 1990), but on the remaining layers we use rectified linear units (ReLus) to reduce computation time Agostinelli et al., 2014). As our model seeks to solve a classification problem, we use categorical cross entropy for the loss function, which we seek to reduce using the Nadam optimizer as this does not require the choice of a learning rate (Dozat, 2016).

To train the model using the five different types of synthetic data, we perform „what is termed "bottleneck learning" in machine learning literature (e.g. Yu and Seltzer (2011)). This is shown in Figure 4A, and comprises of first computing the results from passing our entire dataset through the first five blocks of VGG16, before then training only the fully connected parts of our network (i.e. the classification output). This method is highly efficient as we do not generally wish to update the weights in the convolutional blocks of VGG16, yet passing the data through these blocks is computationally expensive. By passing the data through the convolutional blocks just once, we can then repeat only the relatively inexpensive passes of the data through the fully connected parts of our network as we update the weights contained within these layers.

A common problem of CNNs that are used for classification can be overfitting of the training data, which results in a model that generalises to new data poorly (Krizhevsky et al., 2012). We endeavour to limit this through the use of dropout (Srivastava et al., 2014) before both the 256 and 128 neuron layers, as through randomly removing some connections during each pass of the data through our model, this method aims to ensure that our model is forced to learn more robust representations of the training data. As we 
use synthetic data, we are not limited by the usual cost of collecting labelled data, and therefore are able to generate 20000 unique interferograms that are evenly distributed between classes without the use of data augmentation.

Figure 5 shows the results of training five models with each of the data formats previously discussed. The highest classification accuracy achieved is $\sim 0.95$, which is achieved when the models are trained with either wrapped or unwrapped data repeated across the three input channels. However, it should be noted that the accuracy of the unwrapped phase model takes the full 20 epochs to achieve this performance, which contrasts with the wrapped phase model which shows little change after the eighth epoch. Inclusion of the DEM as the third channel appears to reduce classification accuracy, whilst very low accuracies are achieved in the real and imaginary channel case. We discuss these results in more detail in Section 4, but for the remainder of the paper we choose to work with data that is unwrapped and repeated across the three input channels. We choose this approach as no significant differences are seen between the classification accuracy ultimately achieved with either wrapped or unwrapped data, but the use of unwrapped data may allow for a model to be used with unwrapped time series, and so detect subtle signals produced by low strain rate processes. Additionally, a model that works with unwrapped data may also provide the opportunity to be expanded to locate and classify unwrapping errors automatically. 


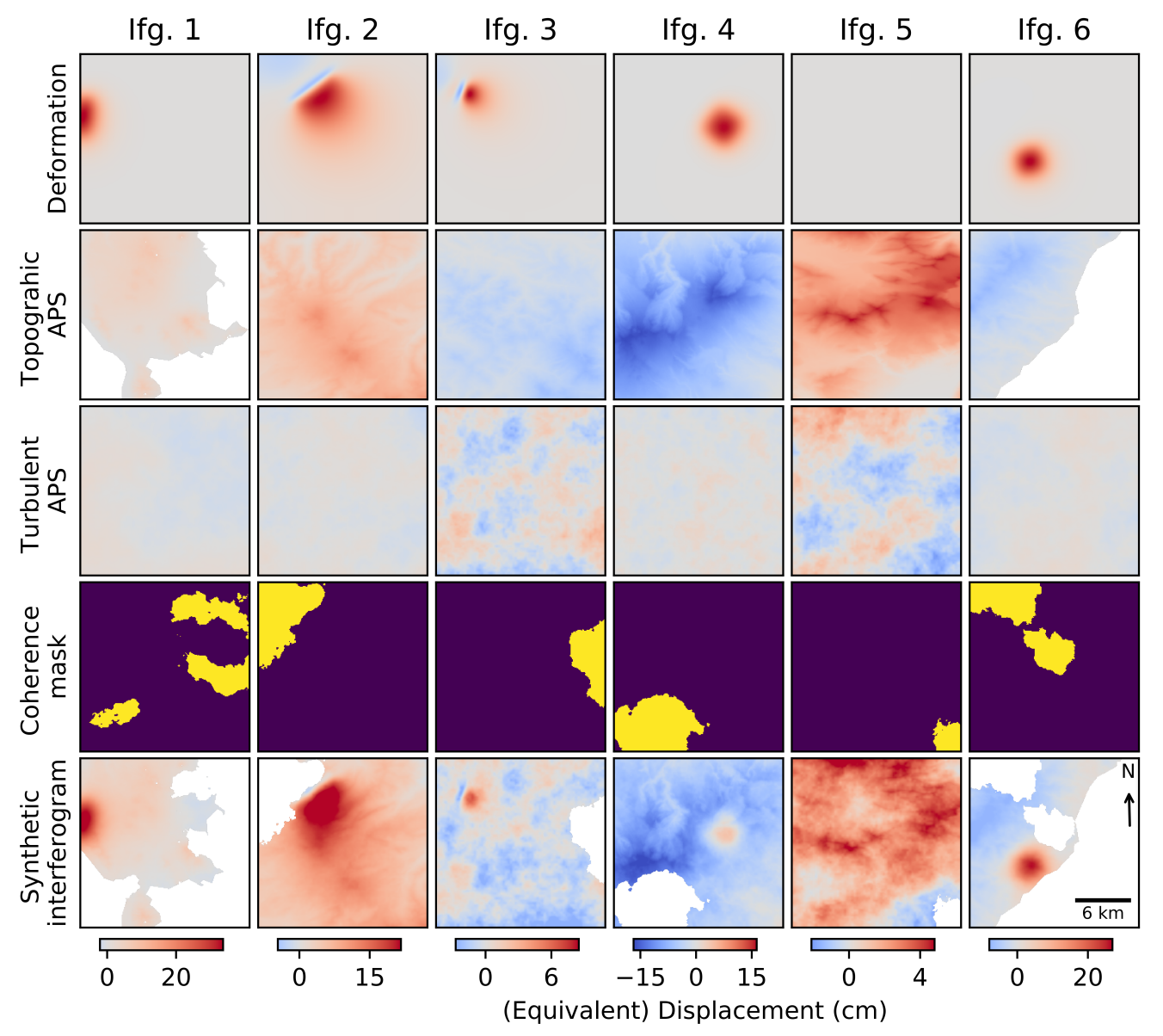

Figure 2: An example of the constituent parts of seven synthetic interferograms. Interferogram 5 does not feature deformation, interferograms 1, 4, and 6 feature deformation due to an sill/point source, and interferograms $2-3$ feature deformation due to an opening dyke. These signals are geocoded and areas of water masked, before being combined with a topographically correlated APS, and a turbulent APS. Areas of incoherence are also synthesised, and these are used to mask the combination of the three signals to create the final synthetic interferograms. 

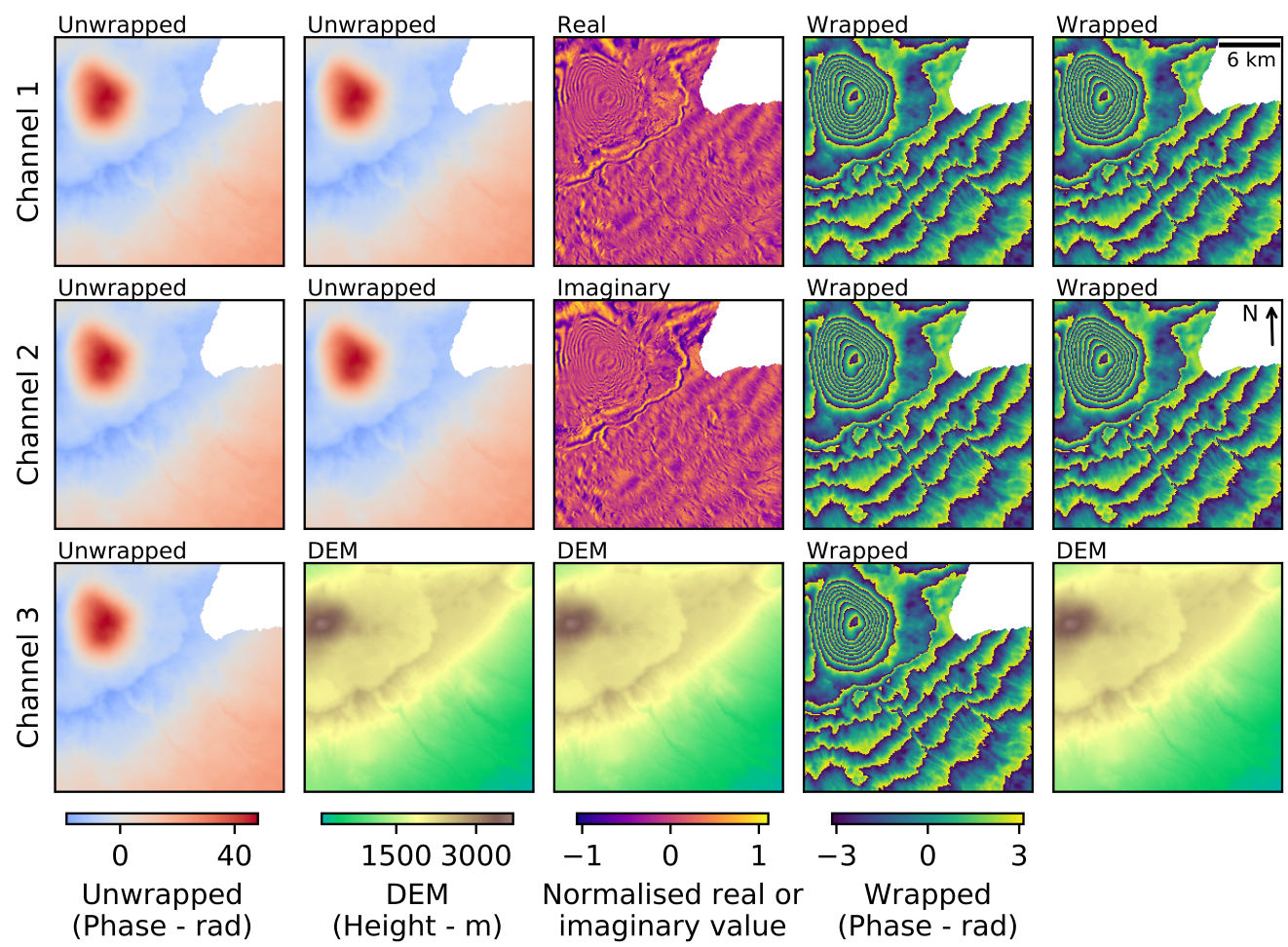

Wrapped

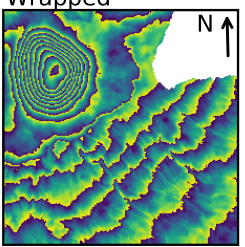

DEM

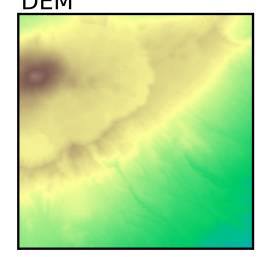

Figure 3: Organisation of an interferogram into three channel form. Columns one and two feature unwrapped data that is repeated, and in column two the DEM is included as the third channel. In column three the real and imaginary elements of the complex values of each pixel of an interferogram occupy channels one and two, whilst the DEM is included in the third. Columns three and four feature wrapped data that is repeated, and in column five the DEM is included as the third channel. 

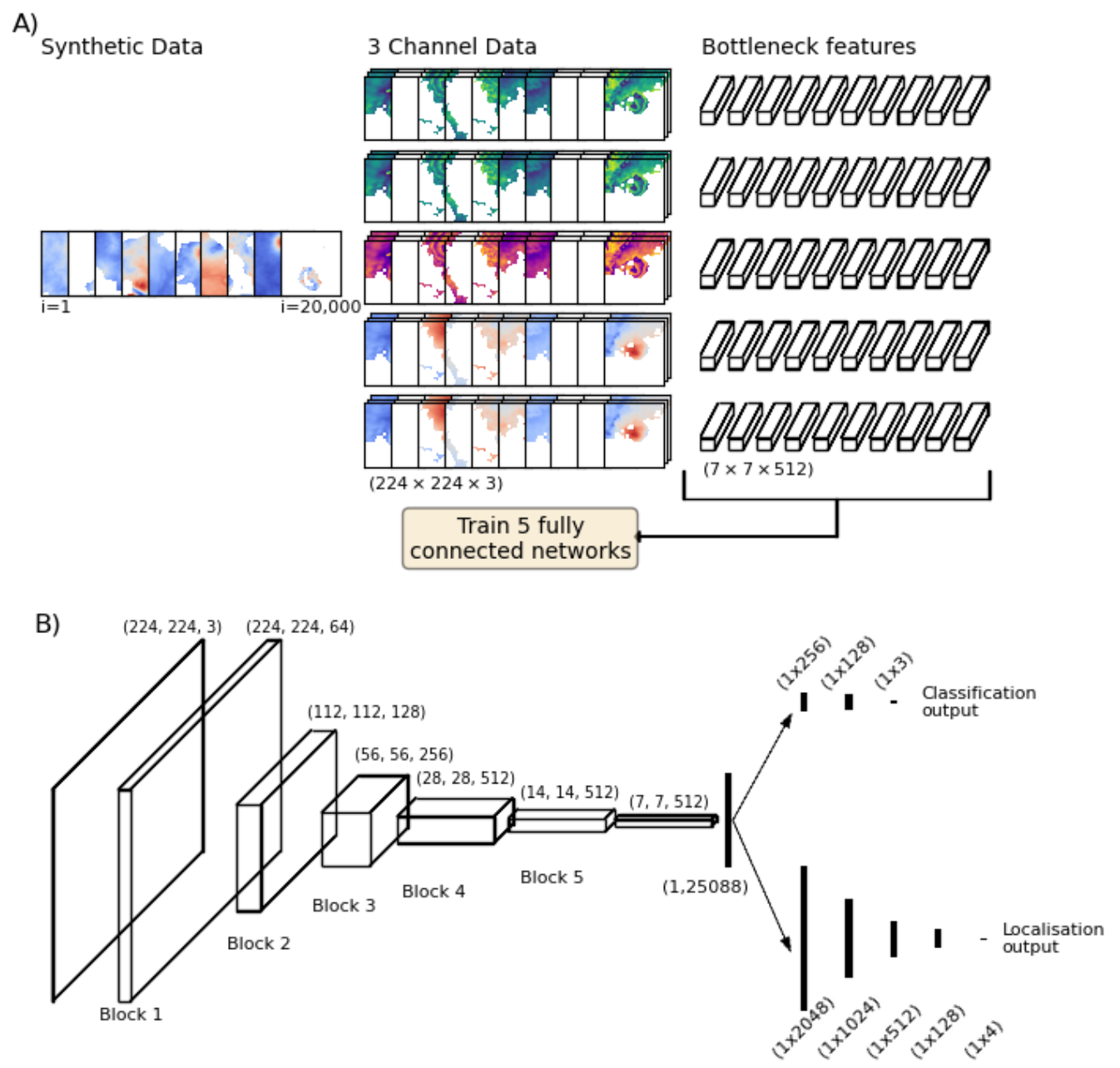

Figure 4: A) Overview of our approach to creating a dataset of synthetic interferograms, arranging these into the five different three channel formats, computing the bottleneck features for each piece of data, and training the fully connected layers of a CNN B)Structure of our classification and localisation CNN. Input interferograms are first passed through the first five convolutional blocks of VGG16 to transform them from size $(224 \times 224 \times 3)$ to size $(7 \times 512)$. These are flattened to create a large fully connected layer featuring 25088 neurons, which is connected to both the upper branch/head, which performs classification, and the lower branch/head, which performs localisation. We find the localisation problem more complex than classification, and consequentially our localisation branch/head features more layers, each with more neurons. The output of the localisation head is a vector

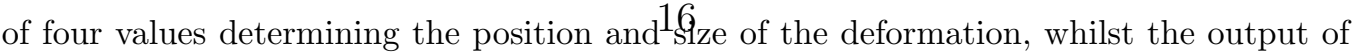
the classification head is a vector of three values that indicate the probability for each class, and sum to one. 


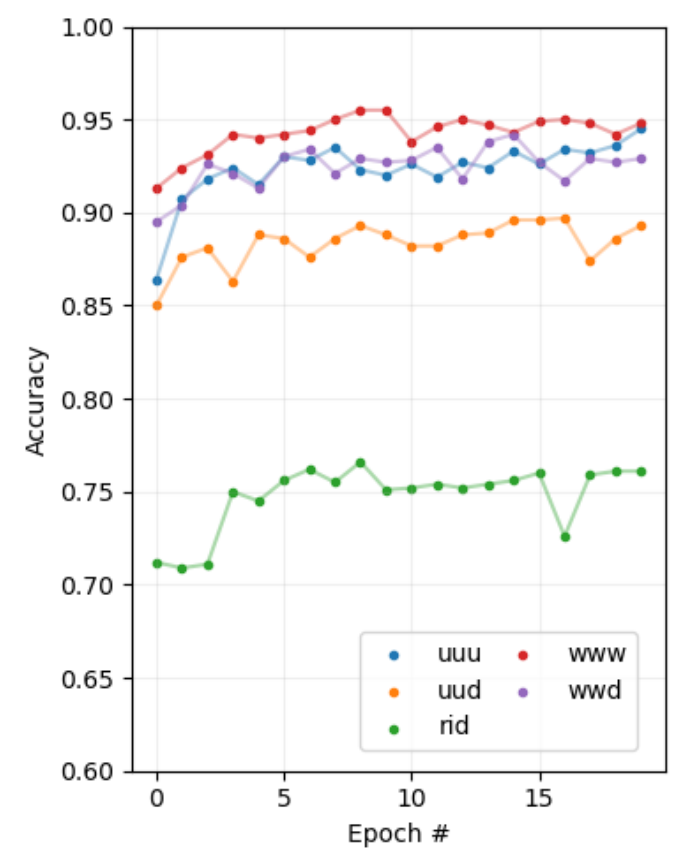

Figure 5: Accuracy of classifying validation data (10\% of the total) during training using three channel data arranged in different formats. "u": unwrapped data, "w": wrapped data, "d": DEM, "r" real component of interferogram, "i": imaginary component of interferogram. Low accuracy is seen for the "rid" data, and in both the wrapped and unwrapped cases inclusion of the DEM in the third channel is seen to degrade classification accuracy. At the end of the 20 epochs of training, only a small difference is seen in accuracy between wrapped and unwrapped data, with both classifying $\sim 95 \%$ of the validation data correctly, though the wrapped phase model is seen to achieve this level of accuracy more quickly (requiring only eight epochs of training). 


\section{Classification and localisation}

\subsection{Using synthetic data}

In the previous section, we demonstrated that, when using VGG16 with convolutional weights learned on ImageNet data, roughly optimal performance for classifying synthetic interferograms is achieved when either the wrapped or unwrapped phase is repeated across the three input channels. We choose to progress with only the unwrapped phase model, as the computational cost of unwrapping is often already met by automatic processing systems (e.g. LiCSAR, Lazeckỳ et al. (2020)), and the development of models that use unwrapped phase may lead to benefits such as the ability to classify and locate unwrapping errors. In this section, we build on the model used to perform classification by adding localisation output. We also endeavour to ascertain if the expense of collecting labelled data can be avoided entirely through the continued use of synthetic data when training our model.

We achieve both classification and localisation through dividing the fully connected section of our model to produce two distinct outputs. One output returns the class of the input data in the manner described in Section 2, whilst the second returns the location and size of any deformation within the scene. In machine learning parlance, models of this type are termed double headed, and we subsequently refer to either of the outputs and their corresponding preceding layers as either the classification head or localisation head. Figure 4B shows the structure of the two heads, and how they diverge after the output of the fifth block of VGG16 has been flattened. The localisation head is structured in a similar manner to the model described in Simonyan and 
Zisserman (2014), in which the model conveys the location of any deformation through outputting a column vector containing four values. Two of these values determine the centre of the deformation pattern and two display its horizontal and vertical extent. Together, these four values can be used to construct a box encompassing a deformation pattern. However, we find that an acceptable level of localisation performance cannot be achieved with a fully connected network with the same complexity as the classification head, and were required to increase both the number and size of layers in the localisation head's fully connected network. Experimentation finds that the simplest model capable of achieving good performance has five layers consisting of 2048, 1024, 512, 128, and 4 neurons.

We use the mean squared error between the predicted location vector and the labelled location vector as our localisation loss function, which we seek to minimise. When using three arc second pixels $(\sim 90 \mathrm{~m})$ with a loss function of this type, a mean square error of 400 pixels would correspond to the localisation being incorrect by around $\sqrt{400}=20$ pixels, or $\sim 2 \mathrm{~km}$. However, when using a double headed network, training is complicated by the fact that the model's overall loss is now a combination of the classification and localisation loss, which must be balanced using a hyperparameter commonly termed loss weighting. We experiment with this hyperparameter, and find that a value of 0.95 for the classification loss and 0.05 for the localisation loss provides a good balance between the two outputs. This value proves suitable as the localisation loss is significantly larger than the classification loss, but by weighting them unequally they then contribute to the overall loss approximately equally. 
An overview of how we trained our model is provided in Figure 6A, but it should be noted that in this step we use only synthetic data. To train our model computationally efficiently, we again employ bottleneck learning, but to improve the performance of our network, we also seek to improve the filters learned within the convolutional blocks. We perform this by changing the style of learning after the 10th epoch, and switch from updating only the fully connected layers, to also including the 5th convolutional block in our updates. However, in contrast to the computationally cheap process of passing only the bottleneck features through the fully connected part of the network, we must now pass the images through both the convolutional and fully connected part of the network. Care is also required with the learning rate when starting the new style of training after the 10th epoch, as too large a learning rate would quickly destroy the finely tuned values in both the convolutional blocks of VGG16, and our fully connected classification and localisation heads. We circumvent this through switching the optimizer to stochastic gradient descent (SGD) and setting the learning rate manually. We find that a value of $1.5 \times 10^{-8}$ does not degrade the performance already gained during training, but still allows for the validation localisation loss to decrease from $\sim 800$ to $\sim 700$ pixels (i.e. a mean error of $\sim 2.6 \mathrm{~km}$ ), and the classification accuracy to increase from $\sim 0.8$ to $\sim 0.85$. This increase in model performance is shown in Figure 6B.

Figure 7 shows the results of applying our trained classification and localisation model to a random selection of the testing data (i.e., $10 \%$ of the data, to which the model was not exposed to during training). In the majority of cases, the classification can be seen to be accurate, and the localisation 
approximately correct. When considering the entire test set of data (i.e. not just the subset shown in Figure 7), the classification accuracy is 0.89 , whilst the localisation loss is $\sim 700$. It should be noted that we could also report the classification loss (0.31), but we believe this is less useful than the accuracy. However, in the localisation case, accuracy is not a meaningful measure of the fidelity of the output, as it is instead a regression problem in which we aim to approximate the correct values, which are continuous in nature. In a manner similar to that reported for the validation data, the localisation loss (mean squared error) of $\sim 700$ pixels corresponds to a mean error of $\sim 2.6 \mathrm{~km}$ (when using three arc second pixels).

\subsection{Application to real data}

Whilst the model described in the previous section achieved good performance when classifying and locating deformation in synthetic interferograms, for use in automatic detection algorithms we require our CNN to work with Sentinel-1 data. These data are of particular importance for volcano monitoring, as the European Space Agency's data policy ensures that Sentinel-1 data are available quickly and at no cost, whilst the low revisit times ensure that the majority of sub-aerial volcanoes are imaged at least every 12 days. To test our model with Sentinel-1 data, we apply our CNN to a collection of 52 interferograms for which we have performed the time consuming task of labelling both the class and location of deformation within them. However, in some examples assigning a single class to a complex deformation pattern is difficult, and we instead assign what we deem the dominant class to be, whilst expecting that the network should assign some probability to other 


\section{A)}
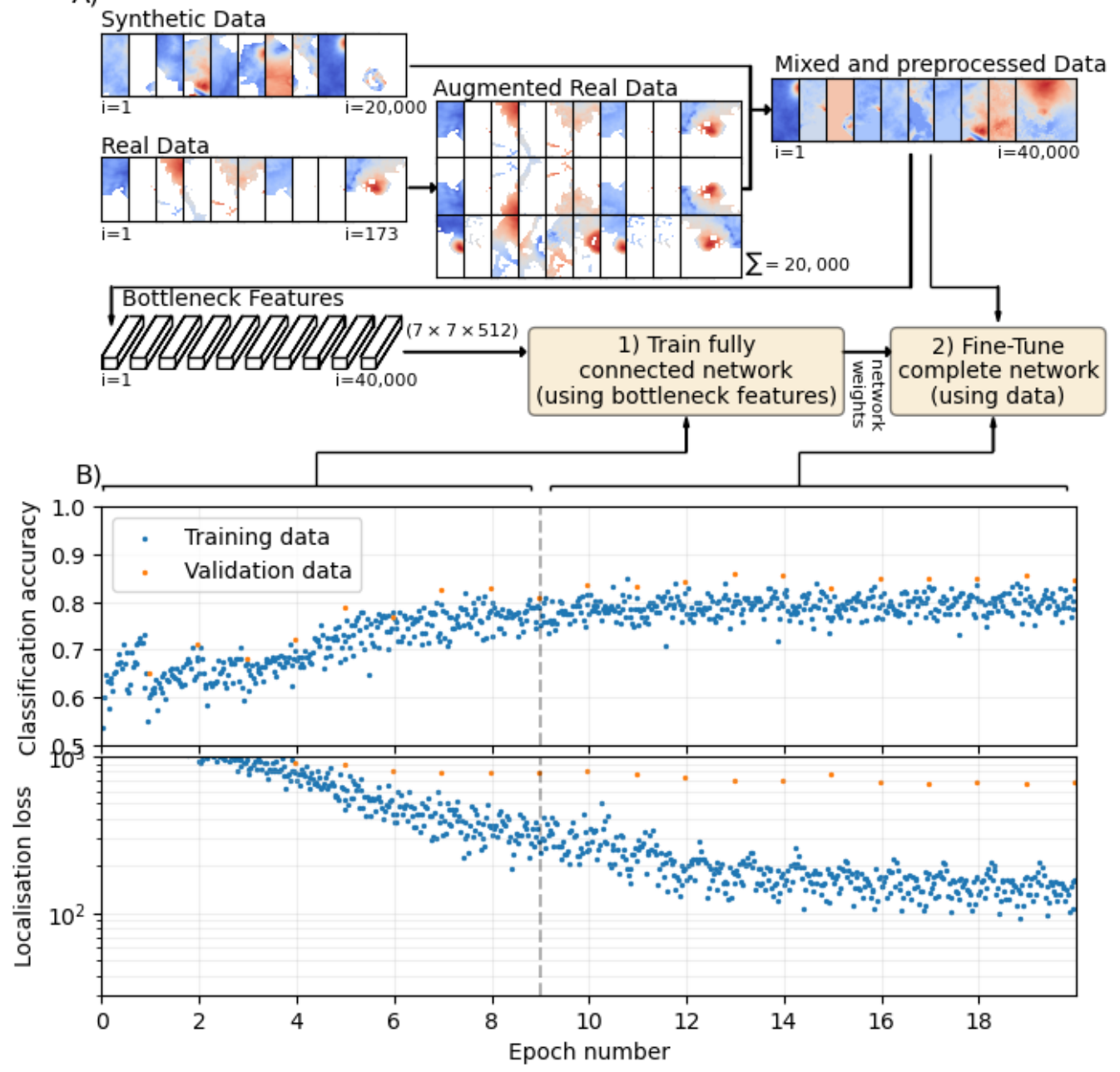

Figure 6: A) Overview of our approach to training our model, in which bottleneck features are used to train only the fully connected network, before the original data are used to fine tune the 5th convolutional block and the fully connected network B) Summary of training the two headed model with synthetic data. The upper plot shows the accuracy of the classification head, whilst the lower plot shows the loss function for the localisation head. To the left of the vertical dashed line bottleneck learning occurred, and to the right traditional learning occurred. This extra learning stage allows the localisation loss for the validation data to decrease from $\sim 800$ to $\sim 700$, and for the classification accuracy of the validation data to increase from $\sim 0.80$ to $\sim 0.85$. 

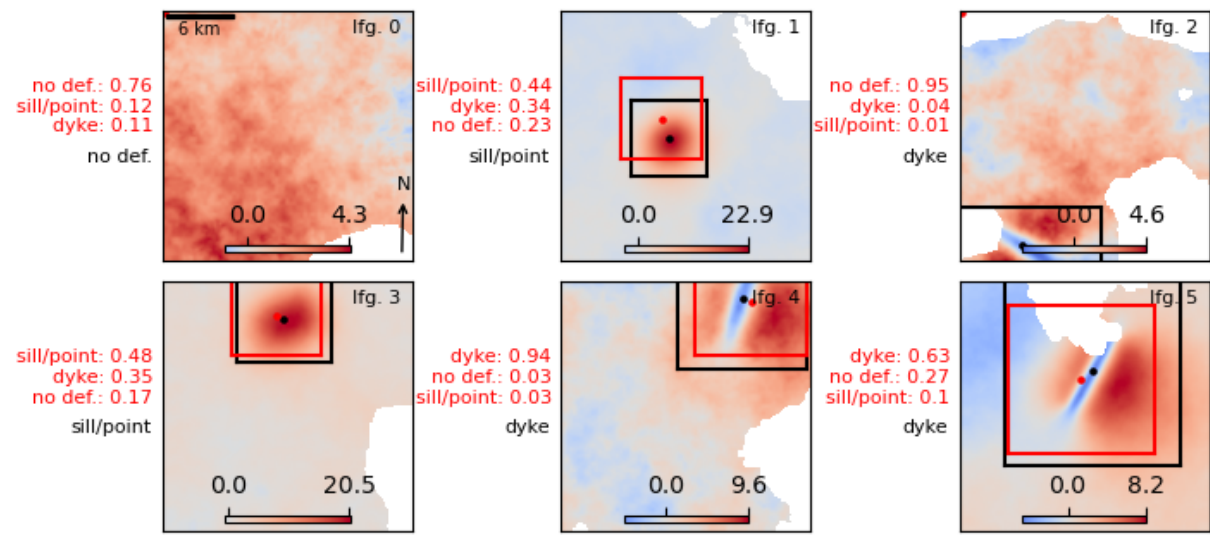

Figure 7: Results of our classification and localisation CNN on the (synthetic) testing data. Deformation units are centimetres, black class labels and location boxes were generated from the synthetic data and encompass all areas with over $5 \mathrm{~cm}$ of deformation, whilst red depicts those predicted by the CNN. As the model outputs a probability for each label, these are included as decimals for each of the predicted classes. Inspection of the results shows that in all but one of the randomly chosen cases, the localisation is broadly correct, and the classification is correct. Interferogram 2, which is classified incorrectly, features a relatively strong turbulent APS (seen as the spatially correlated noise) and a deformation pattern that extends into an area of incoherence, which may explain the misclassification. 
classes. This is most evident in interferograms seven, nine and ten of Figure 8 that span the 2015 eruption of Wolf Volcano (Galapagos, Ecuador), in which signals were attributed to both the deflation of a sill and the opening of a dyke (Novellis et al., 2017; Xu et al. 2016).

The interferograms used come from either a collection of time series that were created by the authors of this study, or by the LiCSAR automatic interferogram processor (https://comet.nerc.ac.uk/COMET-LiCS-portal/), and feature the volcanoes Campi Flegrei, Agung, Wolf, Sierra Negra, and Alcedo. We filtered the interferograms with a Goldstein filter (Goldstein and Werner, 1998), unwrapped using SNAPHU (Chen and Zebker, 2001), and masked pixels with an average coherence below 0.7. For the Galapagos volcanoes (Wolf, Sierra Negra, and Cerro Azul), deformation is visible in some of the 12 day interferograms, but the deformation signal at Campi Flegrei is more subtle, and we are required to manually create interferograms with temporal baselines of $24 / 36 / 48 / 60$ days in order for the deformation to be visible in a single interferogram. The deformation signal at Agung was attributed to the opening of a dyke (Albino et al. 2019), but due to the short lived nature of this event, is only visible in a relatively small number of the "daisy chain" of short temporal baseline interferograms. To increase the number of interferograms available, we again produce a selection of 24/36/48/60 day interferograms that span the event.

Figure 8 shows the results of applying our trained classification and localisation model to a quasi-random selection of Sentinel-1 interferograms. Interferograms such as Interferogram 3 show a very clear inflation signal at Sierra Negra, and are correctly classified by the CNN ("sill/point"), whilst the lo- 
calisation is broadly correct. Other promising results include the labelling of the three Wolf coeruptive interferograms (seven, nine and ten) as containing a sill ("sill/point"), which is also localised well. However, some interferograms are poorly classified, such as the subtle signal seen in interferogram zero. The divergent nature of our CNN's two heads also leads to outputs that show disagreement between them. Interferogram 11 demonstrates this, in which it is correctly classified as containing no deformation, but features an incorrect localisation output.

Considering the entire real testing dataset, the classification accuracy is 0.65 , whilst the localisation loss is $\sim 2017$. We discuss the results of this model more fully in Section 4, but in the following section we seek to improve the performance of our model through the inclusion of real data during the training stage.

\subsection{Augmentation of training data with the VolcNet database of Sentinel-1 data}

To increase the performance of our model further, we seek to incorporate real data into the training. We do this through revisiting the time series that were used to generate the test dataset of 52 interferograms (Section 3.2), and labelling a further 173 interferograms which we use for training, whilst retaining the original set for further testing. This number was chosen as it was the largest that could be created from several time series that were readily available and, whilst we acknowledge that more labelled real data is likely to improve our model, the creation of a significantly larger database remains outside the scope of this paper. Additionally, the use of 

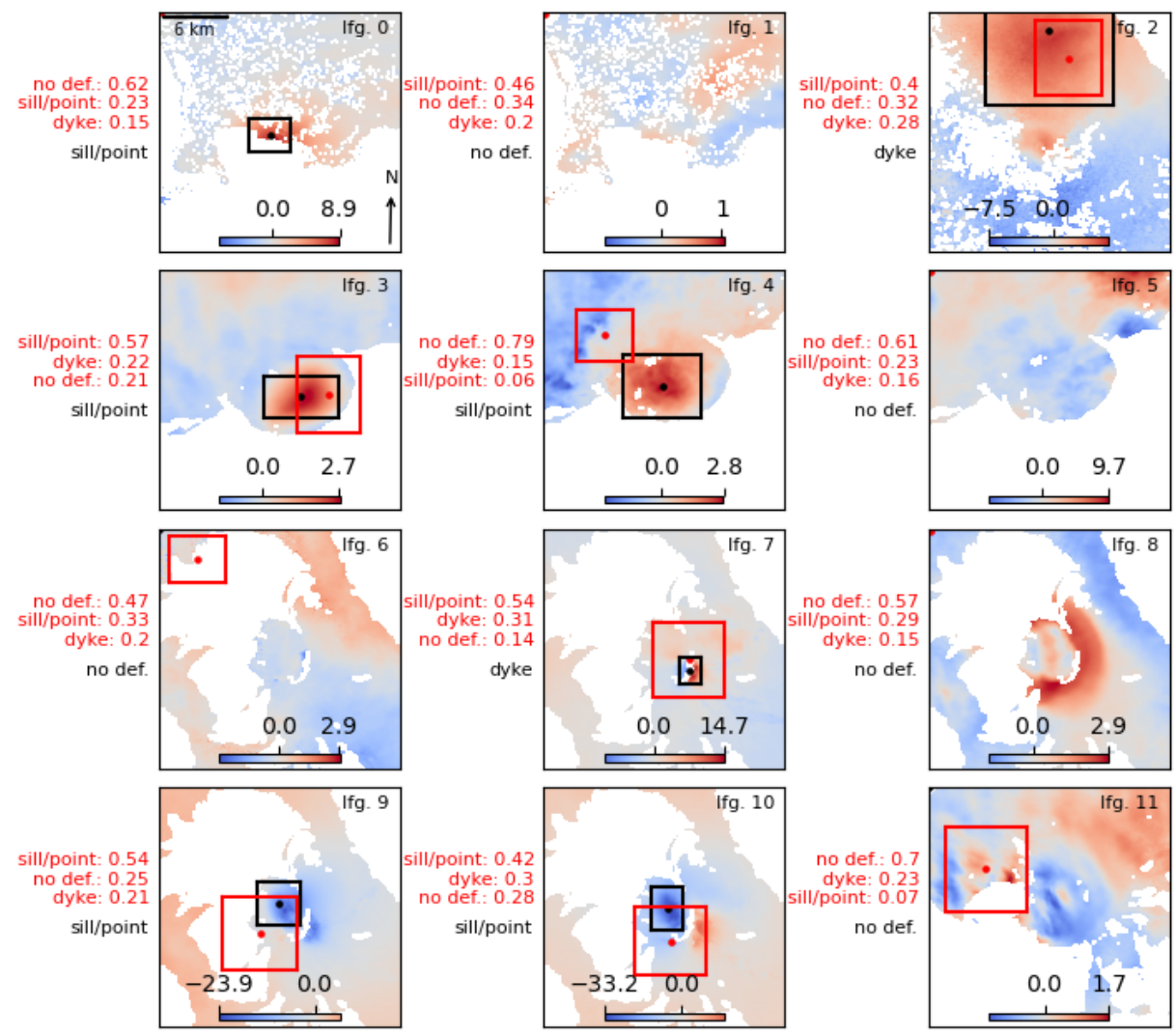

Figure 8: Results of our classification and localisation CNN on our testing set of Sentinel1 interferograms when the CNN has been trained on synthetic data only. The labelling convention is as per the previous figure (n.b. deformation is in centimetres), but labels in black were manually created. Inspection of these results show that they vary between both the label and localisation being broadly correct (e.g. 3, 10), the localisation correct but the label incorrect (e.g. 2), the label correct but the localisation incorrect (e.g. 6), and both the label and localisation incorrect (e.g. 4). Interferograms $0-1$ feature Campi Flegrei, 2 features Agung, 3-5 feature Sierra Negra, 6-10 feature Wolf, and 11 features Cerro Azul. 
a relatively small number of real data allows for greater care to be taken in their labelling, and so reduce the probability of labelling errors occurring. We also make the combined database of $173+52=225$ training and testing data and their labels freely available via GitHub, and term this database VolcNet: https://github.com/matthew-gaddes/VolcNet. However, 20000 synthetic interferograms were used to train the previous model, and the inclusion of 173 new interferograms is unlikely to impact the model significantly as these could still be classified poorly with minimal increase in the loss function. We therefore apply data augmentation, which involves creating random flips, rotations, and translations of the interferograms to extend our set of real training data to feature 20000 unique, though often highly correlated, Sentinel-1 interferograms. With the exception of including real data, we train our model in the same computationally efficient manner as described in the previous section, which we show schematically in Figure 6B.

Figure 9 shows the results of applying our CNN to the same set of test interferograms used in Section 3.2. Inspection shows greatly improved localisation, with very small errors for interferograms zero, two and three. In this selection of interferograms, false positives are not seen (i.e. cases of "no deformation" that are labelled as dykes and sills), but several cases of false negatives are seen, such as interferograms 4, 7, 9, and 10 (i.e. cases of dykes and sills that are labelled as "no deformation". The misclassification of interferogram 4 may be explained through the relatively low signal-to-noise ratio of the deformation signal (i.e. in contrast to interferogram 3), whilst interferograms 7, 9, and 10 feature complex signals that span the 2015 eruption of Wolf and were attributed to both changes in the volume of a sill, and 
propagation of magma to the surface (Xu et al., 2016). As the model was not trained on data that contained multiple deformation signals, the errors seen when this situation is encountered suggests that further work may be needed to incorporate more complex deformation patterns that better reflect the processes that occur at volcanoes.

Considering the entire real testing dataset, performance has now increased, and the classification accuracy has risen to 0.83 , whilst the localisation loss has decreased to 522. Table 1 compares the two models in a more detailed manner by considering the classification accuracy and localisation loss for each class of interferogram. As this is our best performing network, we name it VUDL-NET-21 ("Volcanic Unrest Detection and Localisation NET, 2021), and make all the code required to train it freely available on GitHub: https://github.com/matthew-gaddes/VUDLNet_21

\section{Discussion}

From the analysis performed in Section 2 we conclude that the incorporation of a DEM into our CNN could not be achieved through the relatively simple step of using it as one channel in multichannel data. This is likely because the weights in the first five convolutional blocks of our model were transferred from VGG16 and, as VGG16 was trained using natural images, inputs which are broadly similar across all three channels are required. It should be noted that we rescaled our training data to lie in the same range as the data that VGG16 was trained on (described further in Section 2), and therefore the lack of similarity across channels we refer to is not due to different mag- 

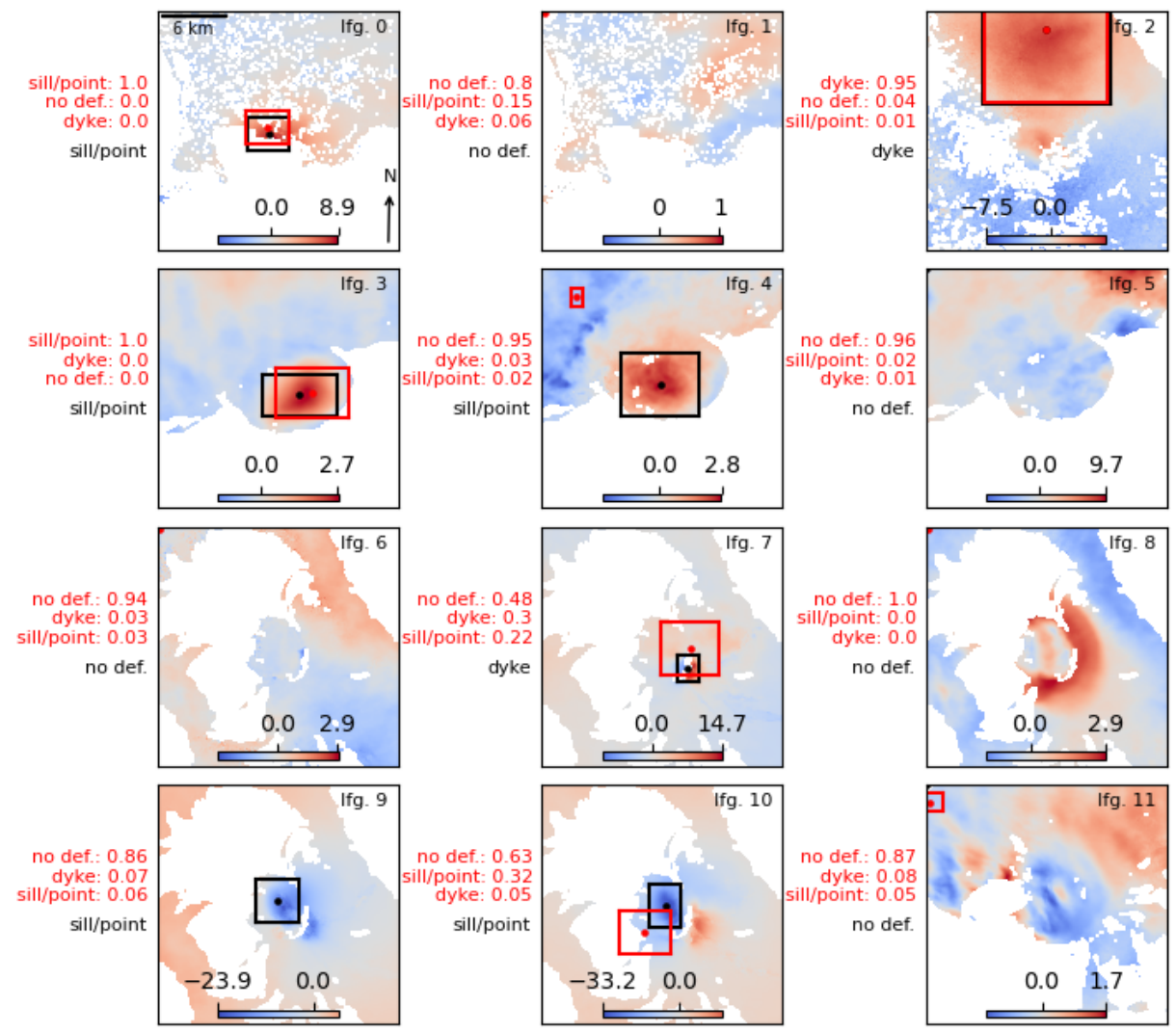

Figure 9: Results of our classification and localisation CNN on our testing set of Sentinel1 interferograms after incoporating real data into the training. The labelling convention and interferograms are as per Figure 8 . This model can be seen to outperform the CNN trained only on synthetic data, with improved classification and localisation. However, several errors remain; e.g., interferogram 4 features a comparatively subtle uplift signal in comparison to others that preceded the 2018 eruption of Sierra Negra and is classified as "no deformation" by the model, whilst the complex co-eruptive signal of interferogram 9 is not located or classified accurately. 


\begin{tabular}{l|ll} 
Classification Accuracy $[0-1]$ & Synthetic & Synthetic and Real \\
\hline Dyke (3) & 0.00 & 0.67 \\
Sill/Point (17) & 0.47 & 0.82 \\
No deformation (32) & 0.81 & 0.84 \\
Combined (52) & 0.65 & 0.83
\end{tabular}

\begin{tabular}{l|ll} 
Localisation Loss (pixels) & Synthetic & Synthetic and Real \\
\hline Dyke (3) & 702 & 100 \\
Sill/Point (17) & 3366 & 579 \\
No deformation (32) & 1423 & 531 \\
Combined (52) & 2017 & 522
\end{tabular}

Table 1: Summary statistics for CNNs trained either with synthetic data, or with synthetic and real data. Combined refers to the complete set of testing data. For both cases, the models can be seen to achieve good accuracy when classifying interferograms that contain either no deformation or deformation due a sill or point source, but to misclassify interferograms that contain deformation due to an opening dyke (accuracies of 0.00 and 0.67). Significant reduction in localisation loss is also seen for interferograms that contain no deformation (1423 to 531 pixels $^{2}$ ), suggesting that the inclusion of real data improves the model's ability to refrain from interpreting atmospheric signals as the location of deformation. 
nitudes, but rather, different spatial patterns. However, an approach where the weights within the convolutional blocks of a classification and localisation model were trained from scratch, may easily allow for the incorporation of extra data in the different input channels. Should this approach not be feasible, information such as the DEM may be best incorporated through the use of a two input model, in which one set of convolutional filters are applied to the phase information, whilst a second is applied to the DEM. These two networks could then be merged at the fully connected stage, in much the same way as our fully connected model diverges into two outputs. Should this be successful, it may also provide a method to add further inputs to a model, such as those outputted by a weather model, which may reduce false positives due to occurrences such as a strong topographically correlated APS. However, training the weights of a model from scratch and exploring more complex multi-input model architectures remains beyond the remit of this study.

The results presented in Figure 8 show that a model trained only with synthetic data is able to classify and locate deformation signals in Sentinel-1 data. However, it is only successful in cases with particularly clear deformation patterns, and in cases with more subtle signals generally erroneously resorts to labelling these as not containing deformation. It is possible that both of these limitations may be overcome through the use of more realistic synthetic data, as our result suggests that our current methodology does not describe processes well enough to be used without real data. The generation of more realistic deformation patterns may be achieved through steps such as more intelligent sampling of the parameters used in the forward models used 
to generate the deformation patterns, the use of different types of deformation models such as penny-shaped cracks (Fialko et al. 2001) or point/Mogi sources (Mogi, 1958), and the superposition of multiple deformation patterns in a single interferogram such as was observed prior to the 2005 eruption of Sierra Negra (Jónsson, 2009). The generation of more realistic atmospheric signals could be achieved through increasing the complexity of synthetic data, such as through the use of phase-elevation ratios that are non-linear or spatially variable, or through using data from different sources. Interferograms that image regions with little deformation could be used to increase the complexity of the set of "no deformation" data, or combined with synthetic deformation patterns to produce more complex semi-synthetic data.

The results presented in Figure 9 show the benefit of incorporating real data. However, much scope for improvement remains, with several classification and localisation errors visible in this figure. The majority of the localisation errors are either in cases in which the deformation signal is slight (e.g. interferogram four of Figure 9, or in interferograms that span the 2015 eruption of Wolf volcano. In the former case, it is natural for a threshold in the signal to noise ratio to exist below which a method is not able to identify the signal of interest, and these interferograms appear to represent that. In the latter case, the interferograms in question contain complex deformation patterns due to both the opening of a dyke and the removal of magma from a sill below the caldera (Novellis et al., 2017), and the inclusion of either real of synthetic training data that contains multiple deformation patterns may alleviate this shortcoming.

The divergent nature of the two heads (classification and localisation) of our 
network also allows for discrepancies between their outputs. This is seen in interferogram 10 of Figure 9, in which the localisation head produces a broadly correct output, but the signal is incorrectly labelled as "no deformation", although with a relatively low confidence. However, we postulate that it may be possible to avoid errors of this type by using more complex model architectures. Models such as YOLO (Redmon et al., 2016) produce bounding boxes and classifications in one step, and have the added bonus of being able to work with images that contain multiple signals. If successfully applied to interferograms, a model of this complexity may avoid the discrepancy errors we encounter, and be able to handle interferograms that contain multiple deformation patterns. In the case that multiple signals do exist in a single interferogram, we do not envisage these to be difficult to label as it is likely that these would be considered interesting events by the scientific community and therefore be the subject of detailed study (e.g. the multi-signal interferograms used in this study are analysed in detail in $(\mathrm{Xu}$ et al., 2016)).

Our approach to localisation avoids the need for repeated classification using a sliding window approach, and allows for our network to reason using the entire image. Whilst this approach is beneficial in terms of advancing the state-of-the-art towards that of a human interpreter, one caveat remains in that building a network that is able to utilise large interferograms can be complex. In our model, we use pixels of three arc second size and, with an input size of $224 \times 224$, the resulting model is able to "see" an approximately $20 \mathrm{~km}$ square around a volcano. If we wish to proceed at this resolution, our model's visual field could be increased through changing the input size to 
around $400 \times 400$ which would not impact our ability to use VGG16's filters (or convolutional blocks), but would increase the size of the first layer of the fully connected part of our network.

At present, an input with side length 224 is reduced to a feature map with side length 7 (shown in Figure 4) which, combined with a depth of 512, produces a flattened layer of size $7 \times 7 \times 512=25088$. However, doubling the input side length would double the feature map side length, increasing the flattened layer to a size of $14 \times 14 \times 512=100352$. Whist our model contains millions of free parameters, connecting this layer to a subsequent layer would produce a significant increase in the total, and is likely to require either more ingenuity or more data to be trained successfully. Analysis of the offsets of deformation patterns at volcanic centres by Ebmeier et al. (2018) finds that $8 \%$ of signals are located more than $10 \mathrm{~km}$ from a volcanic edifice, and would therefore be missed by our current model. Future models that wish to perform localisation using a global approach may therefore require slight increases in size in order to capture all signals of interest.

\section{Conclusion}

We find that either wrapped or unwrapped data are approximately equally suited for use with the weights of VGG16's filters trained on ImageNet data, whilst more complex use of the three channel format that these models support degrades performance. However, we expect this will not be the case if the weights within VGG16's filters are trained from scratch, as additional data such as topography should help to separate deformation from noise.. 
We combine the five convolutional blocks of VGG16 with two fully connected networks to perform both classification and localisation, which allows our network to reason using the whole interferogram (i.e. avoiding a sliding window approach), and therefore move a step closer to interpreting InSAR data in a manner similar to a human expert. Additionally, our network is able to differentiate between several different forms of deformation.

To minimise the costly nature of labelling data, we initially train our model using only synthetic data. We find that our model generalises well to some cases of Sentinel-1 data, but errors remain in cases such as subtle deformation signals, or unusual atmospheric signals. We alleviate this problem through the inclusion of a small amount of real data during the training phase, and present a model that is able to both classify and locate deformation within $\sim 50$ interferograms of $\sim 20 \mathrm{~km}$ side length.

\section{Acknowledgments}

This work was supported by the NERC Centre for the Observation and Modelling of Earthquakes, Volcanoes and Tectonics (COMET). M. E. G. was supported by the Natural Environment Research Council (NERC) grant Looking Inside Continents from Space (LiCS, NE/ K011006/1), and by a European Space Agency Living Planet Fellowship. This project has received funding from the European Research Council (ERC) through the EU Horizon 2020 project DEEPVOLC (grant number 866085). The Copernicus Sentinel data were acquired by the European Space Agency (ESA) and were obtained by the authors of this study from the Alaska Satellite Facility 
archive (https://www.asf.alaska.edu/). Figures were prepared in Matplotlib (Hunter, 2007), and all CNN work was carried out in KERAS using the TensorFlow backend.

\section{References}

Agostinelli, F., Hoffman, M., Sadowski, P., Baldi, P., 2014. Learning activation functions to improve deep neural networks. arXiv preprint arXiv:1412.6830 .

Albino, F., Biggs, J., Syahbana, D.K., 2019. Dyke intrusion between neighbouring arc volcanoes responsible for 2017 pre-eruptive seismic swarm at agung. Nature communications 10, 748.

Anantrasirichai, N., Biggs, J., Albino, F., Bull, D., 2019a. A deep learning approach to detecting volcano deformation from satellite imagery using synthetic datasets. RSE , 1-15doi:10.1029/2018JB015911.

Anantrasirichai, N., Biggs, J., Albino, F., Bull, D., 2019b. The application of convolutional neural networks to detect slow, sustained deformation in insar time series. Geophysical Research Letters 46, 11850-11858.

Anantrasirichai, N., Biggs, J., Albino, F., Hill, P., Bull, D., 2018. Application of Machine Learning to Classification of Volcanic Deformation in Routinely Generated InSAR Data. Journal of Geophysical Research : Solid Earth , 1-15doi:10.1029/2018JB015911.

Beauducel, F., Briole, P., Froger, J.L., 2000. Volcano-wide fringes in ers synthetic aperture radar interferograms of etna (1992-1998): Deformation 
or tropospheric effect? Journal of Geophysical Research: Solid Earth 105, $16391-16402$.

Biggs, J., Ebmeier, S.K., Aspinall, W.P., Lu, Z., Pritchard, M.E., Sparks, R.S.J., Mather, T.a., 2014. Global link between deformation and volcanic eruption quantified by satellite imagery. Nature communications 5, 3471 . doi:10.1038/ncomms4471.

Bridle, J.S., 1990. Probabilistic interpretation of feedforward classification network outputs, with relationships to statistical pattern recognition, in: Neurocomputing. Springer, pp. 227-236.

Chen, C.W., Zebker, H.A., 2001. Two-dimensional phase unwrapping with use of statistical models for cost functions in nonlinear optimization. Journal of the Optical Society of America 18, 338-351.

Deng, J., Dong, W., Socher, R., Li, L.J., Li, K., Fei-Fei, L., 2009. Imagenet: A large-scale hierarchical image database, in: Computer Vision and Pattern Recognition, 2009. CVPR 2009. IEEE Conference on, Ieee. pp. $248-255$.

Dozat, T., 2016. Incorporating nesterov momentum into adam .

Ebmeier, S.K., Andrews, B.J., Araya, M.C., Arnold, D.W.D., Biggs, J., Cooper, C., Cottrell, E., Furtney, M., Hickey, J., Jay, J., Lloyd, R., Parker, A.L., Pritchard, M.E., Robertson, E., Venzke, E., Williamson, J.L., 2018. Synthesis of global satellite observations of magmatic and volcanic deformation : implications for volcano monitoring \& the lat- 
eral extent of magmatic domains. Journal of Applied Volcanology , 126doi: $10.1186 /$ s13617-018-0071-3.

Farr, T., Rosen, P., Caro, E., Crippen, R., 2007. The Shuttle Radar Topography Mission. Reviews of Geophysics 45, 1-33. doi:10.1029/ 2005RG000183.1.

Fialko, Y., Khazan, Y., Simons, M., 2001. Deformation due to a pressurized horizontal circular crake in an elastic half-space, with application to volcano geodesy. Geophys. J. Int. 146, 181-190.

Gaddes, M., Hooper, A., Bagnardi, M., Inman, H., Albino, F., 2018. Blind signal separation methods for insar: The potential to automatically detect and monitor signals of volcanic deformation. Journal of Geophysical Research: Solid Earth .

Goldstein, R.M., Werner, C.L., 1998. Radar interferogram filtering for geophysical applications. Geophysical Research Letters 25, 4035. doi:10. 1029/1998GL900033.

Hanssen, R.F., 2001. Radar interferometry: data interpretation and error analysis. volume 2. Springer Science \& Business Media.

He, K., Gkioxari, G., Dollár, P., Girshick, R., 2017. Mask r-cnn, in: Proceedings of the IEEE international conference on computer vision, pp. 2961-2969.

Hunter, J.D., 2007. Matplotlib: A 2d graphics environment. Computing In Science \& Engineering 9, 90-95. 
Jónsson, S., 2009. Stress interaction between magma accumulation and trapdoor faulting on Sierra Negra volcano, Galápagos. Tectonophysics 471, 36-44. URL: http://linkinghub.elsevier.com/retrieve/pii/ S0040195108003880, doi:10.1016/j.tecto.2008.08.005.

Krizhevsky, A., Sutskever, I., Hinton, G.E., 2012. Imagenet classification with deep convolutional neural networks, in: Advances in neural information processing systems, pp. 1097-1105.

Lazeckỳ, M., Spaans, K., González, P.J., Maghsoudi, Y., Morishita, Y., Albino, F., Elliott, J., Greenall, N., Hatton, E., Hooper, A., et al., 2020. Licsar: An automatic insar tool for measuring and monitoring tectonic and volcanic activity. Remote Sensing 12, 2430.

Mogi, K., 1958. Relations between the eruptions of various volcanoes and the deformations of the ground surfaces around them. Bulletin of the Earthquake Research Institute 36, 99-134. doi:10.1016/j.epsl.2004. 04.016 .

Novellis, V.D., Castaldo, R., Luca, C.D., Pepe, S., Zinno, I., Casu, F., Lanari, R., Solaro, G., 2017. Source modelling of the 2015 Wolf volcano ( Galápagos ) eruption inferred from Sentinel 1-A DInSAR deformation maps and pre-eruptive ENVISAT time series. Journal of Volcanology and Geothermal Research 344, 246-256. doi:10.1016/j.jvolgeores.2017. 05.013

Okada, 1985. Surface deformation due to shear and tensile faults in a half-space. International Journal of Rock Mechanics and Min- 
ing Sciences Geomechanics Abstracts 75, 1135-1154. URL: http:// linkinghub.elsevier.com/retrieve/pii/0148906286906741, doi:10. 1016/0148-9062(86)90674-1.

Redmon, J., Divvala, S., Girshick, R., Farhadi, A., 2016. You only look once: Unified, real-time object detection, in: Proceedings of the IEEE conference on computer vision and pattern recognition, pp. 779-788.

Rémy, D., Chen, Y., Froger, J.L., Bonvalot, S., Cordoba, L., Fustos, J., 2015. Revised interpretation of recent insar signals observed at llaima volcano (chile). Geophysical Research Letters 42, 3870-3879.

Simonyan, K., Zisserman, A., 2014. Very deep convolutional networks for large-scale image recognition. arXiv preprint arXiv:1409.1556 .

Srivastava, N., Hinton, G., Krizhevsky, A., Sutskever, I., Salakhutdinov, R., 2014. Dropout: a simple way to prevent neural networks from overfitting. The Journal of Machine Learning Research 15, 1929-1958.

Sun, J., Wauthier, C., Stephens, K., Gervais, M., Cervone, G., La Femina, P., Higgins, M., 2020. Automatic detection of volcanic surface deformation using deep learning. Journal of Geophysical Research: Solid Earth 125, e2020JB019840.

Valade, S., Ley, A., Massimetti, F., DHondt, O., Laiolo, M., Coppola, D., Loibl, D., Hellwich, O., Walter, T.R., 2019. Towards global volcano monitoring using multisensor sentinel missions and artificial intelligence: The mounts monitoring system. Remote Sensing 11, 1528. 
Xu, W., Jónsson, S., Ruch, J., Aoki, Y., 2016. The 2015 Wolf volcano (Galápagos) eruption studied using Sentinel-1 and ALOS-2 data. Geophysical Research Letters, 9573-9580doi:10.1002/2016GL069820.

Yip, S.T.H., Biggs, J., Albino, F., 2019. Reevaluating volcanic deformation using atmospheric corrections: Implications for the magmatic system of agung volcano, indonesia. Geophysical Research Letters 46, 13704-13711.

Yu, D., Seltzer, M.L., 2011. Improved bottleneck features using pretrained deep neural networks, in: Twelfth annual conference of the international speech communication association. 